\title{
ORGANIZAÇÃO DA DEMANDA E OFERTA DE SERVIÇOS NA ESTRATÉGIA SAÚDE DA FAMÍLIA
}

\section{ORGANIZATION OF SERVICE SUPPLY AND DEMAND OF THE FAMILY CARE STRATEGY}

\author{
Pricila Oliveira Araújo, Marluce Maria Araújo Assis \\ Universidade Estadual de Feira de Santana - UEFS
}

\begin{abstract}
This article aims to discuss about the organization of demands and supply of services in the Family Care Strategy in two scenarios in Bahia, Brazil. Qualitative study carried out with 102 interviewees (health care workers, managers and users) in two cities of Bahia, 2013. The techniques for collecting data were semi-structured interviews and systematic observations. Data treatment was based on thematic content analysis and took into account supply categories of health care services; programmatic and spontaneous demands. There are two kids of demands in the Family Care Strategy, programmatic and spontaneous. The first focuses on specific groups with guidance of appointment and delimitation in the number of vacancies. We checked out that users complain about difficulties in having a treatment with a physician; and nurses try to meet the demands when physicians cannot do it. Assistance to spontaneous quests was organized as in the following typology: high demands, based on the disease and on necessity of immediate treatment; not-high demands, with situations that can wait for assistance and induced demands, assisted on a specific weekday. The organization of demands in the Family Care Strategy happens through action programming and assistance to spontaneous quests, which shows concernment in assisting all the users who go to the unit. However, service supply is still marked by bureaucracy and has as a focus on the disease or its imminence, and it breaks up care and limits integral assistance.
\end{abstract}

Key words: Family Health; Health Services Needs and Demand; Health Services.

\section{Resumo}

Este artigo tem como objetivo discutir a organização da demanda e a oferta de serviços na Estratégia Saúde da Família, em dois municípios da Bahia, Brasil. Estudo qualitativo, realizado com 91 entrevistados (trabalhadores de saúde, dirigentes e usuários), em dois municípios da Bahia. As técnicas de coleta de dados foram entrevista semiestruturada e observação sistemática. A análise dos dados se baseou na Hermenêutica Crítica e considerou as categorias: oferta de serviços de saúde; demanda programada e demanda espontânea. $\mathrm{Na}$ Estratégia Saúde da Família coexistem dois tipos de demandas, programática e espontânea. A primeira tem foco em grupos específicos, com direcionamentos de agenda e delimitação de vagas. $A$ atenção à busca espontânea se organiza na seguinte tipologia: demanda aguda, baseada na doença e na necessidade de atendimento imediato; demanda não aguda, sustentada por situações que podem aguardar atenção; $e$ demanda induzida, assistida em um dia da semana específico. A organização das demandas na Estratégia Saúde da Familia acontece por meio da programação de ações e da atenção à busca espontânea dos usuários. Entretanto, a oferta de serviços ainda é burocratizada e tem como centro da atenção, a doença ou a iminência da mesma, o que fragmenta o cuidado e limita a integralidade.

Palavras chave: Saúde da Família; Necessidades e Demandas de Serviços de Saúde; Serviços de Saúde 


\section{Introdução}

O Programa Saúde da Família (PSF), criado em 1994, com posterior mudança para a designação Estratégia Saúde da Família (ESF), desde a sua concepção, tem, como princípio, a busca pela reorganização do modelo assistencial até então vigente, curativista e hospitalocêntrico. Por meio de programas de saúde que têm foco em afecções frequentes, a partir do perfil epidemiológico da população, a ESF preconiza que as pessoas acometidas tenham atenção priorizada, através de ações de prevenção de riscos e agravos à saúde, tratamento e reabilitação, com vistas à promoção da saúde. Essa forma de organização foi pensada para substituir os modelos tradicionais de atendimento dos centros de saúde ancorados na queixa-conduta. A organização da demanda, então, passou a configurar o cotidiano do trabalho dos profissionais de saúde, e ainda se mantém predominante no Brasil, sendo caracterizada pela marcação prévia dos atendimentos e pela organização das agendas dos profissionais, segundo tipos específicos de atendimento (por programas ministeriais, e grupos específicos como: criança, adulto, mulher, idoso, entre outros) ${ }^{1}$.

As terminologias demanda e necessidade, com frequência, são utilizadas como sinônimos entre si. Entretanto, conceitualmente, demanda é o pedido explícito que o usuário faz no serviço de saúde, a partir de suas necessidades mais complexas. Necessidades de saúde, por sua vez, significa ter condições satisfatórias de vida; acesso e consumo de tecnologia de saúde capaz de qualificar e prolongar a vida; vínculos afetivos e efetivos entre usuários, equipe e profissional; além de ter as condições de saúde respeitadas e graus crescentes de autonomia ${ }^{2}$. Dessa forma, o conceito de necessidade de saúde é amplo, complexo e subjetivo, e pode ser enunciada pelos usuários nas instituições de saúde por meio da terminologia demanda.

Em meio à valorização da demanda organizada, a busca espontânea do usuário ao serviço de saúde foi secundarizada, vez que não havia consenso sobre como as equipes de saúde deveriam atuar frente aos imprevistos, e qual fluxo de atendimento a pessoa deveria seguir ${ }^{3}$, o que gerava demandas reprimidas e repetidos retornos à unidade de saúde.

Com a Política Nacional de Humanização (PNH), a demanda espontânea passou a ser alvo de discussão e possui as seguintes diretrizes de atuação: a elaboração de projetos de saúde individuais e coletivos para os usuários; o acolhimento e a otimização dos serviços para aumentar a resolubilidade da atenção; a corresponsabilização entre trabalhadores e usuários para a melhoria das condições de saúde; e a promoção de hábitos saudáveis de vida ${ }^{4}$. Nesse contexto, era preciso rever as formas de abordagem dos sujeitos, de forma que a demanda enunciada fosse acolhida, escutada, problematizada, e elaboradas soluções correspondentes ${ }^{3}$.

A oferta de serviços na Estratégia Saúde da Família (ESF) compreende atividades que priorizem a solução dos problemas mais comuns da comunidade, ações focalizadas em grupos vulneráveis e de risco, primeiro atendimento às urgências médicas e odontológicas, e ações individuais e coletivas para a promoção da saúde, prevenção de doenças e danos evitáveis ${ }^{5}$. Assim, busca-se a articulação entre as ações programáticas e a atenção à demanda espontânea, que também deve fazer parte do processo de trabalho das equipes, de modo que seja resolutiva. Os serviços ofertados modulam a demanda e, por isso, esta passa a se organizar mediante ao que é disponibilizado ${ }^{6}$.

A influência de modelos assistenciais que não valorizam a pessoa, mas sim o procedimento, faz com que usuários e trabalhadores racionalizem as demandas à doença, aos cuidados especializados e às tecnologias de alta densidade, centralizando o cuidado no profissional de saúde. Isso faz com que a oferta de serviços e demandas na ESF seja objeto de estudos, ao tratar sobre os motivos pelos quais as pessoas buscam os serviços de saúde $^{6}$ e como os trabalhadores de saúde equacionam as demandas, considerando a humanização das práticas na atenção básica ${ }^{7}$.

Trabalhos internacionais tratam da oferta de serviços e práticas na atenção primária através de formas não tradicionais de organização, no intuito de atender as demandas, tais como: a análise da procura e da oferta de visitas noturnas ${ }^{8}$; em horário estendido e aos finais de semana ${ }^{9}$. Além disso, em regiões de difícil acesso aos serviços de saúde, como áreas remotas e rurais, foram encontradas inovações na organização e prestação de serviços de saúde, com a expansão dos papéis profissionais de enfermeiros e clínicos gerais para atender as necessidades daquelas populações ${ }^{10}$.

$\mathrm{Na}$ perspectiva da demanda, estudo no Brasil analisa como é retratada a atenção à demanda aguda, como esta se articula à demanda programática e quais as visões que 
orientam o atendimento na ESF. Os autores afirmam que o processo de trabalho das equipes é fragmentado, as ações dos profissionais são compartimentadas e não se estabelece articulação entre os dois tipos de demandas ${ }^{11}$. Em outra pesquisa, ao discutir as práticas da enfermeira na ESF, as autoras concluem que as mesmas são orientadas nas ações programáticas e na vigilância à saúde, o que contribui para a qualidade de vida das famílias sob sua responsabilidade ${ }^{12}$.

Entretanto, na construção do estado da arte não foram encontrados estudos que tratassem das demandas que poderiam emergir do território social, e que careciam de organização e intervenções adequadas, no âmbito da ESF. Por isso, pesquisar a organização das demandas e a oferta de serviços de saúde, situando o usuário como centro da atenção, traz inovações a partir deste estudo.

Diante disso, o objetivo deste estudo é discutir a organização da demanda e da oferta de serviços na Estratégia Saúde da Família, em dois municípios da Bahia, Brasil.

\section{Metodologia}

Trata-se de um estudo de abordagem qualitativa, do tipo exploratório e descritivo, realizado em dois municípios da Bahia, sendo um situado na zona de transição entre o Recôncavo e o Semiárido (município um), e o outro localizado no Recôncavo da Bahia propriamente dito (município dois). Foram pesquisadas 11 (onze) Unidades de Saúde da Família (USF), sendo 07 (sete) no município um e 04 (quatro) no município dois.

Os participantes da pesquisa foram divididos em três grupos, sendo eles: 37 usuários, 40 trabalhadores de saúde (não apenas profissionais de saúde, mas todos os trabalhadores que atuam na USF) e 14 gestores, totalizando 91 entrevistados; sendo 51 no município um, e 40 no município dois. Os critérios de inclusão foram: trabalhadores de saúde e gestores com mais de seis meses de atuação na Estratégia Saúde da Família, ou no cargo; e usuários maiores de 18 anos e que residiam no território da unidade pesquisada. E os critérios de exclusão foram: trabalhadores de saúde que estavam de licença médica, licença maternidade, ou de férias, e usuários que utilizavam os serviços da USF com frequência menor que duas vezes ao ano.

Assinala-se que a representatividade dos grupos se expressa a partir da relevância política e das ideias que permeiam um dado momento histórico-social, e esta representação se constitui pelo encontro das ordens cognitiva, sociológica e política $^{13}$. O quantitativo de sujeitos da pesquisa foi definido pela saturação dos dados empíricos, através da repetição dos discursos, e pelo delineamento das informações que trouxeram contribuições significantes ao objeto de estudo. A coleta de dados foi realizada durante o período de outubro de 2012 a janeiro de 2013.

As técnicas de coleta de dados utilizadas foram entrevista semi-estruturada e observação sistemática, constando questões comuns e específicas para os distintos grupos de sujeitos, sistematizadas em roteiro orientador com tópicos sobre sistema de regulação, encaminhamentos, serviços ofertados e atendimento às demandas. As observações foram realizadas nas mesmas unidades cujas equipes foram entrevistadas, e o roteiro constava de tópicos sobre o acesso do usuário ao serviço de saúde e o fluxo de atendimento, tais como, recepção do usuário, marcação de consultas, triagem, encaminhamento, acolhimento, dentre outros. Os contatos com os entrevistados se deram por meio telefônico para agendamento da entrevista, exceto para os usuários que foram selecionados de forma aleatória, no momento da observação.

O tratamento e análise do material empírico pautaram-se na hermenêutica crítica ${ }^{14}$. Esta implica interpretar, relacionar e extrair conclusões em todas as direções ${ }^{13,15}$. O primeiro passo compreendeu a ordenação dos dados, no qual foram realizadas as transcrições e organização desses dados, em cada um dos dois municípios em estudo e, em seguida, agrupamento das entrevistas por grupos de sujeitos. Na classificação dos dados, procedeu-se a leitura flutuante e exaustiva de cada entrevista, identificaram-se as categorias empíricas em relação às ofertas e demandas que emergiram das unidades de saúde da família para serviços de média densidade tecnológica, e realizou-se a síntese vertical de cada uma, o que possibilitou a ideia geral de cada entrevista. Em seguida, uma leitura transversal permitiu apreender os discursos convergentes e complementares dos diferentes grupos de sujeito.

As informações adquiridas com os dados da observação sistemática foram cruzadas e confrontadas com as sínteses das entrevistas. $\mathrm{Na}$ análise final, foram realizadas inferências e interpretações com a base teórica do estudo, sendo elaboradas árvores analíticas e fluxograma analisador. 
O projeto obedeceu às normas para pesquisa com seres humanos e teve aprovação do Comitê de Ética em Pesquisa da Universidade Estadual de Feira de Santana (CAAE no 05549512.2.00000053) e das Secretarias Municipais de Saúde (SMS) de ambos os municípios.

\section{Resultados e Discussões}

Os resultados dos dados empíricos de ambos os cenários demonstraram que a oferta dos serviços de saúde e suas formas de organização da demanda na ESF são baseadas em ações programáticas por grupos específicos, e na procura espontânea por atendimento.

As sínteses dos conteúdos que emergiram dos discursos dos grupos de usuários, trabalhadores e gestores são apresentadas na Figura 1.

Figura 1. Síntese analítica dos dados empíricos (entrevistas) dos usuários, trabalhadores e dirigentes.

\section{OFERTA DE SERVIÇOS NAESTRATÉGIA SAÚDE DA FAMIILIA}

- Atendimento eminentemente agendado e individualizado;

- Organização das agendas realizada a partir da opinião da comunidade. Marcação de consulta para médico obedecendo a critérios de sele ção: microárea, familia ou ordem de chegada; e para enfermeiro, com agendamento diário ou no ato da consulta. O quantitativo de vagas para atendimento é estipulado previamente e varia entre as USF;

- Critérios de atendimento: residir no território da USF; ter agendamento prévio ou uma demanda que deve ser priorizada; obedecer ao quantitativo de vagas;

- Não of erece serviços à noite e aosfinais de sem ana.

ORGANIZAÇÃO DAS DEMANDAS NA ESTRATÉGIA SAÚDE DA FAMÍLIA

\begin{tabular}{|c|c|c|c|}
\hline Demanda Programática & \multicolumn{3}{|c|}{ Demanda Espontânea } \\
\hline -Programa de controle & Aguda & Não aguda & Induzida \\
\hline $\begin{array}{l}\text { hanseniase, hipertensão } \\
\text { arterial, diabetes } \\
\text { mellitus, pré-natal, } \\
\text { planejam ento familiar, } \\
\text { entre outros; }\end{array}$ & $\begin{array}{l}\text {-Situaçõesagudas } \\
\text { comfoco na } \\
\text { doença(febre, } \\
\text { diarréia, dor } \\
\text { abdominal). }\end{array}$ & $\begin{array}{l}\text {-Medicações; } \\
\text {-Curativos; } \\
\text {-Troca de sonda; } \\
\text {-Orientações; } \\
\text {-Situaçőescom foco } \\
\text { nadoença; }\end{array}$ & $\begin{array}{l}\text {-Delimitação de um } \\
\text { dia da semana para } \\
\text { atender demanda } \\
\text { espontânea. }\end{array}$ \\
\hline
\end{tabular}

\section{A oferta de serviços na Estratégia Saúde da Família}

Os serviços de saúde ofertados na ESF dos municípios estudados são organizados de forma a prestar atenção eminentemente individualizada e com agendamento prévio. A organização dos mesmos acontece, principalmente, com base nos programas ministeriais. A organização dos atendimentos por linhas temáticas como saúde da criança, do adulto, do idoso, da mulher, do homem, saúde mental, citada pelos entrevistados, também é delineada pelas ações programáticas. Neste caso, pode haver um ajuste da oferta do serviço, de acordo com a demanda apresentada no território social, e a agenda do profissional de saúde passa a ser organizada com foco na atuação programática.

A robustez dos atendimentos na ESF acontece mediante a organização das agendas dos profissionais de nível superior. A marcação das consultas médicas nos municípios pesquisados é realizada em um dia específico da semana ou do mês, sendo que os critérios de seleção e agendamento são definidos em reuniões comunitárias. Geralmente, segundo os entrevistados, decidem-se pela ordem de chegada, exceto nos casos de urgências e emergência.

Usuários apresentaram queixas relacionadas ao atendimento médico: ter que chegar muito cedo à fila e aguardar muito tempo para ser atendido; médico que atrasa, atende poucas pessoas e não permanece até o fim do turno de trabalho; além da dificuldade de acesso 
a consultas, sendo que, para driblar estas barreiras, os usuários recorriam à rede de relações sociais como mecanismo alternativo, para resolução das demandas. Entretanto, a observação sistemática mostrou que a população, apesar da insatisfação com tal atendimento, permanecia em silêncio e não se queixava para os trabalhadores no ato do cuidado.

Uma das explicações para a não reclamação por parte dos usuários seria a assimetria de poder entre estes e os trabalhadores, que é potencializada pelo modelo assistencial profissional-centrado e pela falta de exercício da cidadania, estabelecida culturalmente, e não percebida enquanto direito constitucional ${ }^{16}$. Observa-se um discurso geral dos usuários de indignação individual, mas não de detecção dos problemas e buscas de soluções coletivas. A oferta de serviços de saúde na ESF é alvo de insatisfação por parte dos usuários que, muitas vezes, não têm suas demandas atendidas e pouco reclamam, ou questionam tal forma de organização.

As representações dos discursos obtidos evidenciam que as demandas na ESF estão ancoradas ao modelo profissional centrado e, por isso, a satisfação das necessidades de saúde está associada ao agendamento da consulta médica e à ausência de enfermidades ${ }^{17}$. Ou seja, quando questionados sobre a oferta de serviços na ESF, os sujeitos se remetiam às dificuldades relativas ao atendimento médico.

No caso do enfermeiro, os usuários relataram facilidades nas marcações para o atendimento com este profissional, já que as consultas poderiam ser agendadas diariamente, ou no fim de cada atendimento poderia ser combinado o retorno, o que parece favorecer a continuidade do cuidado e o vínculo entre profissional e usuário.

\section{A organização das demandas na Estratégia Saúde da Família: foco na atenção programática}

A organização dos serviços estudados é baseada na demanda programática e atende grupos específicos e prioritários, definidos a partir do perfil epidemiológico da população; além de ser caracterizada pela prévia definição de vagas e direcionamentos da agenda dos profissionais para assistir aos usuários cadastrados em programas. Esse tipo de abordagem é importante porque advêm de rastreamento epidemiológico, por meio do qual se identifica determinantes e condicionantes do processo saúde-doença, que interferem no perfil de morbimortalidade da região ${ }^{18}$. Entretanto, entende-se que tal forma de atuação não deve ser exclusiva, à medida que pode reforçar a rigidez das marcações nos serviços, além de limitar a resolubilidade da ESF, ainda que isso não seja buscado ${ }^{19,20}$. Ao não se observar as singularidades dos sujeitos, aqueles que não correspondem a grupos prioritários podem ser excluídos do cuidado, conforme ressaltado pelos trabalhadores entrevistados.

Por isso, apesar do Ministério da Saúde (MS) dispor de protocolos, construídos por consensos científicos, que direcionam a atuação dos trabalhadores da ESF frente aos principais agravos que acometem a comunidade, é preciso que eles sejam utilizados com análise crítica e adaptados à realidade da população ${ }^{21}$. Além disso, é preciso promover encontros, individuais e coletivos que estimulem a criatividade da equipe para que, no confronto com as necessidades sociais e de saúde da comunidade, seja capaz de mediar respostas adequadas focadas na pessoa ${ }^{19}$.

o atendimento programático é predominantemente realizado pelo médico e enfermeiro, todavia, na lógica do acolhimento, a ESF necessita oferecer outros serviços para atender as demandas do usuário sem, no entanto, limitar-se ao atendimento desses profissionais e da queixa em $\mathrm{si}^{19}$. Geralmente, critica-se a categoria médica pela atenção biologicista, porém, enfermeiros também têm reproduzido práticas individualizadas e centradas em procedimentos ${ }^{20}$. A análise das entrevistas e da observação sistemática do presente estudo mostra que, tanto médicos quando enfermeiros, acompanhavam os usuários cadastrados nos programas. Esta forma de organização da demanda diverge de uma outra pesquisa, ao mostrar que a demanda programática era assistida pelo enfermeiro, e a espontânea, pelo médico $^{11}$.

Estudo realizado com enfermeiros identificou que as atividades programáticas foram apontadas como inovação no trabalho da atenção básica e funcionavam como guia para direcionar a atuação dos profissionais junto a grupos específicos, substituindo a organização da oferta de ações centrada na demanda espontânea, que historicamente tem se organizado nos tradicionais centros de saúde ${ }^{22}$. A realidade dos municípios estudados mostrou que trabalhadores de diversas categorias valorizavam a organização da demanda programática, a ponto de desqualificarem a atenção à busca 
espontânea, como se esta fosse sinônimo de desorganização no atendimento, com exceção dos gestores, que compreenderam que o cuidado às necessidades momentâneas era importante e, por isso, deveria ser valorizado e realizado com qualidade.

\section{O lugar da demanda espontânea na Estratégia Saúde da Família}

A terminologia utilizada pelas equipes para a atenção à demanda espontânea variou entre equipes e trabalhadores que, normalmente, chamavam de atendimento extra, atendimento de urgência e emergência, ou mesmo demanda espontânea. A análise dos relatos mostra que, em ambos os municípios, esse tipo de atendimento era organizado segundo alguns critérios de horário ou dias, e de quantitativo de vagas.

Os critérios eram estipulados pelas equipes de saúde que, no caso do município um, definiam que $o$ atendimento à demanda espontânea tinha média de quatro vagas e deveria ser realizado ao final das consultas agendadas. Já no município dois, a lógica da organização diferenciava-se do município um, na medida em que se especificava um dia da semana (sexta-feira) para esse atendimento. Assim, em ambos os casos, se esses critérios fossem cumpridos de forma rígida poderia haver demandas reprimidas, pois pessoas ficariam sem atendimento. Além do mais, as demandas, por vezes, se manifestam de forma imprevisível. Apesar dos critérios, os entrevistados ressaltaram que os casos urgentes, ou que não podiam aguardar atendimento, eram conduzidos com prioridade. Os dados da observação sistemática corroboraram esta informação.

A iniciativa para trabalhar com esse tipo de demanda é recente e surgiu a partir da Política Nacional de Humanização, por meio da proposta de Acolhimento ${ }^{4,19}$. Até então, a lógica da organização da demanda a partir dos grupos específicos, priorizados pela ESF, desvalorizava a busca espontânea do usuário, pois, segundo o MS, não havia recomendações sobre como a equipe deveria atuar frente aos imprevistos frequentes e inevitáveis no cuidado à saúde. Atualmente, o MS preconiza a integração entre as ações programáticas e a demanda espontânea.

A demanda espontânea representa a autopercepção do usuário ao identificar a necessidade de buscar o serviço de saúde e, geralmente, é de resolução imediata ${ }^{18}$. material empírico mostra que o atendimento não agendado é realizado quando existe urgência no caso, e as situações de emergências são encaminhadas às policlínicas ou hospitais. Outro estudo também evidencia que a demanda espontânea, na atenção primária, tem sido orientada pela lógica do atendimento da demanda aguda, o qual é centrado no profissional de saúde e na resolução de queixas $\operatorname{orgânicas~}^{11}$. De forma geral, a procura por esse tipo de atendimento pode ser justificada pelo desejo de encaminhamento para especialistas ou de obter medicação gratuita ${ }^{23}$.

Ademais, vale ressaltar que as demandas do usuário perpassam por processos biológicos e representações culturais sobre a saúde e a doença; pelas experiências subjetivas dos sujeitos sobre o sofrimento; pela referência social e jurídica dos direitos e deveres relativos à saúde; pela percepção do que é ofertado nas unidades de saúde e de como esses elementos interagem entre $\mathrm{si}^{24}$. Assim, um homem de 50 anos que vai a primeira vez ao serviço de saúde, por exemplo, necessita ser acolhido pelos trabalhadores, independente de enunciar uma demanda considerada urgente ou não.

Compreender que a demanda espontânea deve ser direcionada apenas pela gravidade do caso, pode proporcionar a desconsideração das questões não orgânicas e subjetivas do usuário. É como se houvesse uma limitação da capacidade de escuta da equipe, que também perde a oportunidade de intervir em situações de risco e vulnerabilidade. Assim, quando o trabalhador não consegue objetivar as queixas dos usuários a questões biológicas, o profissional tende a desqualificá-las e a não reconhecê-las como legítima aos serviços de saúde ${ }^{11}$.

O usuário também define de variadas formas e graus o que é necessidade de saúde e como pode apresentá-la ao serviço em forma de demanda $^{3}$. Existem intervenções que necessitam da continuidade do cuidado, o que significa dizer que assistir à demanda espontânea nem sempre é possível em único encontro, mas, a partir dele, a equipe pode sensibilizar o usuário para a importância do retorno à unidade, identificar a verdadeira demanda e elaborar formas de intervenção e cuidado.

Às vezes pode haver convergência entre o olhar técnico do profissional e a queixa enunciada pelo usuário, entretanto, isso pode não acontecer e, mesmo assim, a demanda deve ser acolhida, escutada e problematizada a fim de encontrar soluções correspondentes ${ }^{3}$. Afinal, identificar a demanda não é tarefa fácil, uma vez 
que é fruto de um processo de negociação entre os sujeitos envolvidos, que são históricos e subjetivos.

Na Figura 2 é apresentado o fluxograma de representação das demandas dos usuários na ESF. A atenção à busca espontânea se organiza na seguinte tipologia: demanda aguda, não aguda e induzida.

Figura 2. Fluxograma de representação das demandas dos usuários na ESF.



A demanda aguda seria aquela em que o usuário apresenta uma queixa que necessita de cuidado no momento, como febre alta, dor intensa, etc. Nos municípios estudados, foi observado que casos emergenciais eram tratados com prioridade, apesar dos murais informativos comunicarem quantidade de vagas delimitada para esses atendimentos. Já outra pesquisa mostra que mesmo usuários com febre ou dor, antes de serem atendidos, eram submetidos ao crivo do número de vagas e horários determinados, o que contrariava as práticas de humanização e acolhimento ${ }^{18}$.

A demanda não aguda é caracterizada por situações que não precisam de atenção imediata, e que podem aguardar atendimento ao final dos usuários que estão marcados; e demanda induzida, realizada em um dos municípios de estudo, é delineada a partir da especificação de um dia da semana para assistência aos casos espontâneos.

Ao passo que a equipe reconhece a importância da atenção à demanda espontânea, é insuficiente restringir tal escuta a momentos específicos, ou mesmo elaborar um conjunto de barreiras no atendimento para o usuário que não se ajustar às normas estabelecidas pela equipe. Quaisquer que sejam as ações organizacionais, os arranjos para atender à demanda real de cada usuário são fundamentais ${ }^{3}$. Caso contrário, pode haver burocratização do serviço e demandas reprimidas.
A organização do processo de trabalho das ESF não está estruturada a partir da lógica do acolhimento $^{3}$. Isso faz com que o encontro dos sujeitos, que deveria ser caracterizado por atos cuidadores, é marcado pela mordaça do modelo assistencial praticado, pelo silêncio do usuário e autoritarismo do profissional ${ }^{21}$, pela fragmentação do cuidado e desconsideração da integralidade ${ }^{6}$.

De modo geral, a lógica que deve nortear a atuação dos profissionais na USF é que usuários com atividades de rotina agendadas sejam acolhidos e direcionados para o atendimento, evitando esperas desnecessárias; que as situações imprevistas, inerentes à vida, sejam organizadas de maneira que a equipe possa compreendê-las e intervir; e que os trabalhadores que escutam primeiramente as demandas espontâneas sejam capazes de analisá-las, dialogar com outros colegas e ofertar cuidados, visando à resolubilidade da atenção ${ }^{3}$.

\section{Conclusões}

Os dados empíricos mostram que nas USF coexistem os dois tipos de demanda, a organizada e a espontânea, o que demonstra preocupação não somente com os grupos prioritários, mas também com os usuários que recorrem ao serviço com demandas diversas, sem agendamento prévio.

O atendimento programático direciona a atuação dos trabalhadores para as afecções mais frequentes na comunidade, a partir do rastreamento epidemiológico, e é organizado de forma que, em cada turno da semana, seja assistido um grupo diferenciado, delimitado por médicos e enfermeiros. Entretanto, ainda existe forte queixa dos usuários relacionada às dificuldades na obtenção da consulta médica na USF, o que revela a influência do modelo médico centrado. Usuários não relatam barreiras nos agendamentos para as consultas de enfermagem, e o enfermeiro tenta resolver as demandas dos usuários, quando há inviabilidade do atendimento médico.

A organização da oferta de serviços, em ambos os municípios, possibilita ampliar a atenção à demanda espontânea. Porém, salvo as situações urgentes (demanda aguda), a organização é burocratizada, com um dia da semana específico (demanda induzida), ou ao final de cada turno de atividades (demanda não aguda), com quantidade de vagas delimitadas e critérios baseados na doença ou iminência da 
mesma. As equipes compreendem que o atendimento de emergência e urgência não é foco da ESF, entretanto, nesses casos, devem ser realizados acolhimento, atenção inicial e encaminhamentos, quando necessário. A coexistência de ambas as formas de atuação constitui-se em elementos que podem potencializar a resolutividade na ESF e transformar o modelo de assistência em usuáriocentrado, a partir do acolhimento, escuta qualificada e educação permanente.

\section{Referências}

1. Assis MMA, Cerqueira EM, Nascimento MAA, Santos AM, Jesus WLA. Atenção Primária à Saúde e sua articulação com a Estratégia Saúde da Família: construção política, metodológica e prática. Revista APS 2007 jul/dez; 10(2): 189-99.

2. Cecílio LCO. As Necessidades de Saúde como Conceito Estruturante na Luta pela Integralidade e Equidade na Atenção em Saúde. In: Pinheiro R, Mattos RA, organizadores. Os sentidos da integralidade na atenção e no cuidado à saúde. Rio de Janeiro: Uerj/ Ims: Abrasco; 2001. p. 113-25.

3. Ministério da Saúde (BR), Secretaria de Atenção à Saúde, Departamento de Atenção Básica. Acolhimento à demanda espontânea: queixas mais comuns na Atenção Básica. Brasília (DF): Ministério da Saúde; 2011a.

4. Ministério da Saúde (BR), SecretariaExecutiva, Núcleo Técnico da Política Nacional de Humanização. HumanizaSUS: Política Nacional de Humanização: a humanização como eixo norteador das práticas de atenção e gestão em todas as instâncias do SUS. Brasília (DF): Ministério da Saúde; 2004.

5. Ministério da Saúde (BR). Portaria $\mathrm{n}$ 은 2.488, de 21 de outubro de 2011. Aprova a Política Nacional de Atenção Básica. Brasília (DF): Ministério da Saúde; 2011b.

6. Franco TB, Merhy EE. A Produção Imaginária da Demanda e o Processo de Trabalho em Saúde. In: Pinheiro $R$, Mattos RA, organizadores. Construção social da demanda: direito à saúde, trabalho em equipe, participação e espaços públicos. 2a a ed. Rio de Janeiro: Cepesc/Uerj: Abrasco; 2010. p.185-95.

7 Junges JR, Barbiani R, Fernandes RBP. $O$ discurso dos profissionais sobre a demanda e a humanização. Saude Soc 2012 Jul/Mar; 21(3):686-97.
8. Giuffrida A, Gravelle H. Inducing or restraining demand: the market for night visits in primary care. J Health Econ 2001 Sep; 20:755-79.

9. Morgan $\mathrm{CL}$, Beerstecher HJ. Satisfaction, demand, and openinghours in primary care: an observational study. Br J Gen Pract 2011 Aug; p.498-597.

10. Mitton C, Dionne F, Masucci L, Wong S, Law S. Innovations in health service organization and delivery in northern rural and remote regions: a review of the literature. Int J Circumpolar Health 2011 Out; 70(5):460-72.

11. Faria RC, Campos EMS. Demanda Espontânea na Estratégia de Saúde da Família: uma análise dos fatores que a influenciam e os desafios na reorientação do modelo assistencial do SUS. Rev APS 2012 abr/jun; 15(2):148-57.

12. Nascimento $M$, Nascimento MAA. Prática da enfermeira no Programa de Saúde da Família: a interface da vigilância da saúde versus as ações programáticas em saúde. Ciênc. saúde coletiva 2005 Abr/Jun; 10(2):333-45.

13. Minayo MCS. O desafio do conhecimento: pesquisa qualitativa em saúde. 12 ed. São Paulo: Hucitec, 2010. 407p.

14. Gadamer Hans-Georg. Verdade e método. Traços fundamentais de uma hermenêutica filosófica. Tradução de Flávio Paulo Meurer. 3 ed. Petrópolis: Editora Vozes; 1999.

15. Assis MMA, Jorge MSB. Métodos de análise em pesquisa qualitativa. In: Santana JSS, Nascimento MAA, organizadoras. Pesquisa: métodos e técnicas de conhecimento da realidade social. Feira de Santana: Uefs; 2010. p.139-59.

16. Soratto J, Witt RR, Faria EM. Participação popular e controle social em saúde: desafios da Estratégia Saúde da Família. Physis Revista de Saúde Coletiva 2010; 20(4):1227-43.

17. Franco FA, Hino $P$, Nichiata LYI, Bertolozzi MR. A compreensão das necessidades de saúde segundo usuários de um serviço de saúde: subsídios para a Enfermagem. Esc Anna Nery 2012 Jan/Mar; 16(1):157-62.

18. Santana ML. Demanda espontânea e planejamento estratégico situacional no Programa Saúde da Família de Pindamonhangaba. Rev Bras Med Fam Comunidade 2011 Abr/Jun; 6(19):133-41.

19. Tesser CD, Campos GWS, Poli Neto P. Acolhimento, (des)medicalização social e gestão do cotidiano em equipes de saúde da família. In: Tesser CD, organizador. Medicalização Social e 
Atenção à Saúde no SUS. São Paulo: Hucitec; 2010. p.131-50.

20. Santos AM, Giovanella L, Mendonça MHM, Andrade CLT, Martins MIC, Cunha MSC. Práticas assistenciais das Equipes de Saúde da Família em quatro grandes centros urbanos. Ciênc. saúde coletiva 2012 Out; 17(10):2687-702.

21. Malta DC, Merhy EE. O percurso da linha do cuidado sob a perspectiva das doenças crônicas não transmissíveis. Interface (Botucatu) 2010 Set; 14(34):593-606.

22. Silva AMN, Mandú ENT. Abordagem de necessidades de saúde no encontro assistencial de trabalhadores e usuários na Saúde da Família. Texto Contexto Enferm 2012 Out/Dez; 21(4):73947.

23. Santos TVC, Penna CMM. Demandas cotidianas na atenção primária: o olhar de profissionais da saúde e usuários. Texto Contexto Enferm 2013 Jan/Mar; 22(1):149-56.

24. Camargo Júnior KR. Das Necessidades de Saúde à Demanda Socialmente Construída. In: Pinheiro R, Mattos RA, Organizadores. Construção Social da Demanda: direito à saúde, trabalho em equipe, participação e espaços públicos. 2aed. Rio de Janeiro: Cepesc/Uerj: Abrasco; 2010. p.93-103. $\mathrm{CNPq}$

*Pesquisa financiada pelo Edital Universal

\section{Endereço para Correspondência}

Universidade Estadual de Feira de Santana - UEFS

Avenida Transnordestina, $\mathrm{s} / \mathrm{n}$ - Novo Horizonte CEP 44036-900 - Feira de Santana - Bahia

e-mail: poaraujos@gmail.com

Recebido em 10/03/2017

Aprovado em 05/09/2017

Publicado em 30/11/2017 